\title{
Estimación del valor añadido bruto, dependencia espacial y datos de panel: Evidencia en el caso de los municipios de la Región de Murcia *
}

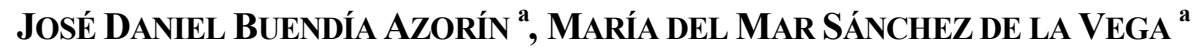 \\ a Universidad de Murcia, Facultad de Economía y Empresa, Campus de Espinardo, 30100 Murcia, \\ España.E-mail: jdbuen@um.es, marvega@um.es
}

\begin{abstract}
RESUMEN
El objetivo de este trabajo es proponer una metodología para la estimación de la macromagnitud Valor Añadido Bruto por habitante $\left(\mathrm{VAB}_{\mathrm{pc}}\right)$ en el ámbito geográfico municipal. El procedimiento utilizado consiste en estimar un modelo de panel que incluye el filtro espacial de Griffith $(1996,2000)$ para capturar la dependencia espacial en todo el periodo analizado. El análisis de regresión entre el $\mathrm{VAB}_{\mathrm{pc}}$ de cada provincia y un conjunto de indicadores genera los coeficientes que se aplicarán a los valores de los indicadores municipales, lo que permite estimar (predecir) de forma robusta el $\mathrm{VAB}_{\mathrm{pc}}$ de los municipios de la Región de Murcia.
\end{abstract}

Palabras Clave: Métodos Semiparamétricos, Datos de Panel, Distribución espacial.

\section{Estimation of Gross Value Added, Spatial Dependence and Panel Data: Evidence in Municipalities of Región of Murcia Case}

\begin{abstract}
The aim of this work is to propose a statistical methodology in order to estimate the macromagnitud Gross Value Added per capita $\left(\mathrm{GVA}_{\mathrm{pc}}\right)$ in the local geographical area. The procedure proposed consists in estimating a panel model which includes Griffith's spatial filtering $(1996,2000)$ to capture the spatial dependence in the whole analyzed period. The relationship between the $\mathrm{GVA}_{\mathrm{pc}}$ of each province and a set indicators generates the coefficients that will be applied to the values of the municipal indicators, which allows foretell efficient estimates of the $\mathrm{GVA}_{\mathrm{pc}}$ of the municipalities of the Region of Murcia.
\end{abstract}

Keywords: Semiparametric Methods, Panel Data Models, Spatial Distributions.

Clasificación JEL: C14, C33, R12

\footnotetext{
* Este trabajo presenta resultados preliminares en el marco de un proyecto de investigación financiado por el Consejo Económico y Social de la Región de Murcia. Los autores agradecen los valiosos comentarios y sugerencias realizados por dos evaluadores anónimos.
}

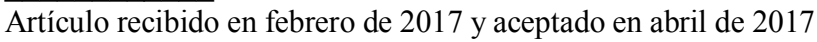

Artículo disponible en versión electrónica en la página www.revista-eea.net, ref. ə-35210 


\section{INTRODUCCIÓN}

La disponibilidad de macromagnitudes económicas de renta y actividad en los diferentes ámbitos territoriales debería considerarse una información básica al alcance de cualquier ciudadano. El hecho de que en España no existan estadísticas oficiales de renta y actividad en el ámbito intrarregional municipal, junto con la necesidad de conocer la situación económica y social de los diferentes territorios locales para la planificación de un desarrollo equilibrado en cada uno de ellos, ha generado un especial interés en el ámbito académico e institucional como lo prueba el importante desarrollo de trabajos destinados a proporcionar estas estadísticas en el ámbito local.

En general, para estimar macromagnitudes en el ámbito municipal se han utilizado dos métodos alternativos: directo e indirecto. El método directo realiza la estimación a partir de la medición de las variables que componen la macromagnitud por lo que requiere una gran cantidad de información estadística $\mathrm{y}$ de recursos materiales y financieros. Esto incluye la realización de encuestas o censos y unos requerimientos de estadísticas básicas muy elevados y un alto coste económico que con frecuencia garantizan un resultado muy fiable. Sin embargo, en algunas situaciones su elevada fiabilidad es cuestionada como consecuencia de la limitación que supone la existencia de importantes bolsas de economía sumergida, lo que imposibilita la correcta medición de la actividad. Por otra parte, los elevados recursos económicos y de tiempo que requiere su aplicación (elaboración, tratamiento y explotación de encuestas) lo convierten en un procedimiento en muchos casos inviable para las instituciones públicas o privadas interesadas.

Por su parte, el método indirecto basa la estimación de la macromagnitud en el análisis de regresión entre la variable dependiente y un conjunto de indicadores. Aquí, adquiere especial relevancia la forma funcional elegida y la capacidad explicativa de los indicadores disponibles que están relacionados con la variable dependiente. Un aspecto que no ha sido considerado en una gran mayoría de trabajos y que no se debe obviar en el tratamiento de los datos disponibles es la posible existencia de dependencia espacial, lo que exigirá la utilización de las técnicas econométricas adecuadas que permitan o bien incorporarla al modelo o eliminarla. Otro aspecto relevante de la estimación es la muestra seleccionada, es decir, se trabaja con datos transversales, con datos temporales o se utilizan conjuntamente ambos mediante datos de panel. El empleo de datos transversales implica que se toman datos para un mismo momento del tiempo de diferentes territorios, bajo el supuesto implícito que el comportamiento de la variable dependiente con respecto a las independientes es idéntico en los diferentes ámbitos de agregación espacial (provincias, comarcas, municipios). El empleo de datos temporales establece la construcción de una serie histórica de datos del ámbito espacial analizado. En esta relación se determina el comportamiento de 
la variable explicada en el ámbito espacial agregado, suponiendo que la relación entre la variable dependiente y las independientes es similar en el ámbito espacial agregado y en cada una de las unidades espaciales. Por último, la utilización de datos de panel permite complementar las dos dimensiones tiempo y espacio, obteniendo resultados más consistentes al integrar las dinámicas espaciales y temporales. En particular, las estructuras de datos de panel espacial permiten incorporar la heterogeneidad espacial o los mecanismos de dependencia espacial. No obstante, desde cualquiera de las tres perspectivas se procede a la estimación de la ecuación de regresión con los datos provinciales y los coeficientes así obtenidos en la ecuación se aplican a los valores de los indicadores municipales, lo que permite estimar la renta de los municipios. Este planteamiento desemboca en el problema de la unidad de área modificable, en su doble vertiente del problema de escala y el problema de agregación. El primero consiste en determinar cuál es el nivel espacial óptimo para estudiar una variable, ya que las relaciones halladas en un determinado nivel no se mantienen en otros y las conclusiones obtenidas en una escala no son válidas en otra. El segundo, está relacionado con la forma de las observaciones y se conoce con el nombre de "falacia ecológica" que consiste en extrapolar los resultados generados con datos agregados al comportamiento de unidades individuales.

La utilización del procedimiento indirecto presenta la virtualidad de su permanente aplicabilidad en las condiciones actuales de disponibilidad informativa estadística y a un coste muy reducido respecto al método directo. Además, la utilización de indicadores permite corregir la limitación de no registrar sistemáticamente (en el caso de las encuestas) una parte sustancial de actividades que al desarrollarse al margen de la normativa legal existente queda fuera de todo registro estadístico. No obstante, el método indirecto con sus características de menores requerimientos de información que el directo, en general, proporciona resultados cuya fiabilidad está condicionada por las limitaciones del planteamiento metodológico y las hipótesis implícitas en el modelo.

Nuestra propuesta es proporcionar un modelo de estimación del $\mathrm{VAB}_{\mathrm{pc}}$ fácilmente aplicable con la disponibilidad de información a escala provincial y municipal y que garantice unos resultados robustos. Este consiste en la estimación econométrica de un modelo de panel espacial del $\mathrm{VAB}_{\mathrm{pc}}$ en el ámbito provincial utilizando variables explicativas que están disponibles también para el ámbito municipal. Posteriormente se extrapolan los parámetros estimados y se multiplican por los indicadores municipales. Por tanto, se acomete la estimación del VAB por el método de estimación estadística en el que adquiere especial relevancia el proceso de búsqueda y selección de indicadores disponibles en el ámbito provincial y municipal que tienen una relación directa con la variable dependiente. 
El resto del trabajo se estructura de la siguiente manera. En el apartado 2 se realiza un breve recorrido de los antecedentes en el ámbito de estimaciones municipales que permite aproximarnos a las experiencias y resultados de investigaciones similares para extraer las posibilidades y limitaciones de este tipo de trabajos en orden a justificar en mayor medida nuestro enfoque metodológico. El apartado 3 expone la metodología empírica utilizada y describe la técnica de filtrado espacial de los datos aplicada en un modelo de panel. En el apartado 4 se presentan los datos y los principales resultados obtenidos de las estimaciones realizadas del modelo. Finalmente, en el apartado 5 se recogen las principales conclusiones obtenidas de este estudio.

\section{BREVE REVISIÓN DE EXPERIENCIAS EN EL ÁMBITO MUNICIPAL}

Como se ha indicado con anterioridad, en España no existen estadísticas oficiales de renta y actividad en el ámbito municipal ${ }^{1}$ lo que ha propiciado un aumento de los trabajos que tratan de proporcionar estadísticas fiables en el ámbito local. Mayoritariamente estos trabajos han centrado su objetivo en la estimación de la renta bruta disponible de los hogares (RBDH) y, en menor medida, en las macromagnitudes valor añadido bruto $(\mathrm{VAB})^{2}$, renta bruta $(\mathrm{RB})^{3}$ y renta personal $(\mathrm{RP})^{4}$. En efecto, existe una larga tradición en España de realizar estimaciones de la renta disponible municipal ${ }^{5} \mathrm{y}$, con carácter general, en los trabajos pioneros mencionados se realizó el ejercicio de regresión mediante el método de Mínimos Cuadrados Ordinarios (MCO) ignorando la

\footnotetext{
${ }^{1}$ En la mayoría de los países desarrollados también existe esta carencia de información oficial a nivel local, debido fundamentalmente al elevado coste de elaboración. Solo en países como Estados Unidos, Gran Bretaña y Australia se elaboran regularmente estadísticas oficiales sobre renta personal local.

2 Alañón (2001) realizó la estimación del valor añadido per cápita de los municipios españoles en 1991. Por su parte, Enrique (2009) estima el valor añadido bruto de los municipios andaluces en el año 2000.

${ }^{3}$ La Agencia Tributaria (AEAT) en cumplimiento de la Ley de Transparencia y para dar publicidad de la información que consta en las declaraciones tributarias elabora la estimación de la Renta Bruta y la Renta Disponible para los municipios de más de 1.000 habitantes. Actualmente la información está referida a los años 2013 y 2014.

${ }^{4}$ Más recientemente, Ortas-Rico y Honrubia (2014) realizan la estimación de la renta personal de los municipios españoles de más de 5.000 habitantes a partir de microdatos tributarios pertenecientes a la Comunidades Autonómas de Régimen Fiscal Común en el año 2007.

${ }^{5}$ Algunos referentes son los trabajos pioneros de Banesto (1967) que estima la renta municipal de los municipios de España, Sadei (1978) la renta familiar disponible de los concejos de Asturias. Posteriormente, se añaden los de Muns (1971), López y Montes (1974), Castells et al. (1982, 1983) Aparicio et al. (1983), Esteban y Pedreño (1988), Remírez (1991) y Otero y Fernández, A. (1999). En esta línea se encuentran también los de Beyaert et al. (1993) y Buendía et al. (1999 y 2010).
} 
dependencia espacial de los datos, lo que produce estimaciones sesgadas e inconsistentes. Los primeros intentos de incorporación de la dependencia espacial en los modelos de estimación de la renta municipal se producen en los primeros años del presente siglo, en los trabajos de Alañón (2001), Chasco (2003), Chasco y López (2004) y Buendía et al.(2010). Con anterioridad, en la segunda mitad de los años noventa, Vayá et al. (1996), incorporaron los efectos espaciales en el análisis de los resultados de la $\beta$-convergencia regional.

En la Tabla 1 se sintetiza la relación de los principales trabajos clasificándolos según el doble criterio de las macromagnitudes estimadas y del método empleado. La estimación de la renta de los municipios de España se abordó solo en los trabajos de Banesto (Anuario Comercial de España) hasta 1995, Instituto L. R. Klein (Anuario Económico de España) hasta 2013 y en el realizado por Alañón en 2001. Muy recientemente se suman a la estimación de renta de los municipios de España el trabajo de Ortas-Rico y Honrubia disponible para los años 2002 a 2007 y el de la Agencia Tributaria para los años 2013 y 2014. El resto de trabajos tienen un alcance limitado a las comarcas o municipios de su demarcación territorial.

Este trabajo continúa la línea más reciente de los desarrollos de la econometría espacial e incorpora como novedad la utilización de datos de panel como se pondrá de manifiesto en los siguientes epígrafes. En concreto, utilizamos el procedimiento del filtrado semiparamétrico de Griffith (1996, 2000) para capturar la autocorrelación espacial en el marco de un modelo de panel. Este procedimiento descompone la variable estudiada en función de una componente sistemática o no espacial, que es una combinación lineal de las variables explicativas observadas, y una componente espacial formada por una combinación lineal de variables sintéticas que representan la estructura espacial del conjunto de datos. En el caso de datos de panel, el filtrado espacial tiene que realizarse en cada período temporal, y en él pueden intervenir distintos subconjuntos de autovectores según el periodo temporal considerado.

$\mathrm{La}$ elección de la macromagnitud a estimar, el $\mathrm{VAB}_{\mathrm{pc}}$, viene impuesta por las nuevas directrices de publicación del Instituto Nacional de Estadística (INE) en el ámbito territorial provincial (NUTS3, en la terminología de Eurostat) que limita la disponibilidad de información económica a nivel NUTS3 a las macromagnitudes Producto Interior Bruto (PIB) y Valor Añadido Bruto (VAB). Así, en la última serie publicada 2010-2013 base 2010, la información relativa a la Renta Bruta Disponible de los Hogares (RDBH) solo está desagregada a nivel de Comunidades Autónomas (NUTS2). Por el contrario, el VAB sí está disponible a nivel provincial y el INE ha publicado recientemente la serie 20002013 base 2010. Por tanto, según la metodología que proponemos, la variable que es susceptible de estimación es la renta producida por habitante $\left(\mathrm{VAB}_{\mathrm{pc}}\right)$ mediante el método indirecto. 
Tabla 1

Estimaciones de renta en el ámbito municipal

\begin{tabular}{l|l|l}
\hline \multirow{2}{*}{ Variable } & \multicolumn{2}{c}{ Metodología ${ }^{(a)}$} \\
\cline { 2 - 3 } & \multicolumn{1}{c}{ Econometría tradicional } & Econometría espacial \\
\hline \multirow{2}{*}{} & Banesto (1967) & Chasco (2003) \\
& Muns (1971) & Chasco y López (2004) \\
& López y Montes (1974) & Buendía et al. (2010) \\
& Sadei (1978) & \\
& Castells et al. (1982) & \\
& Aparicio et al. (1983) & \\
& Caja de Ahorros de Zaragoza, Aragón y la Rioja (1984) \\
& Esteban y Pedreño (1988) & \\
RBDH & Remírez (1991) & \\
& Beyaert et al. (1993) & \\
& Instituto L.R Klein (1995) & \\
\hline VAB & Otero y Fernández (1999) & Alañón (2001) \\
\hline RP & Buendía et al. (2000) & \\
RB & Enrique (2009) & \\
\hline
\end{tabular}

a) Se utilizan métodos de estimación indirecta en la gran mayoría de estos trabajos, exceptuando el realizado por Sadei que combina el método directo y el indirecto.

b) Utilizan los microdatos tributarios de las CC.AA. de Régimen Fiscal Común para estimar la Renta Personal de los municipios españoles de más de 5.000 habitantes. Último año disponible 2007.

c) Utiliza la información tributaria del IRPF de las CC.AA de Régimen Fiscal Común para estimar la Renta Bruta y la Renta Disponible de los declarantes del impuesto en los municipios de más de 1.000 habitantes. En la medida que se agregan los diferentes rendimientos declarados en el impuesto para obtener la magnitud puede considerarse una estimación directa. La renta personal bruta incluye los rendimientos del trabajo, capital mobiliario, capital inmobiliario, rentas de actividades económicas, otras rentas, ganancias patrimoniales netas y rentas exentas. La renta disponible se estima descontando a la renta bruta la cuota líquida resultante del impuesto y las cotizaciones sociales y derechos pasivos a cargo del trabajador consignados en su declaración. Último año disponible 2014.

Fuente: Elaboración propia.

\section{EL FILTRADO SEMIPARAMÉTRICO DE AUTOCORRELACIÓN ESPACIAL: EL ENFOQUE DE LOS VECTORES PROPIOS}

Las técnicas más habituales para el análisis de datos que presentan autocorrelación espacial utilizan modelos que especifican directamente una estructura espacial, siendo los más frecuentes los modelos autorregresivos espaciales ${ }^{6}$.

Un enfoque alternativo consiste en utilizar la técnica de filtrado espacial de Griffith $(1996,2000)$, que es la que aplicamos en este trabajo ${ }^{7}$. El procedimiento

\footnotetext{
${ }^{6}$ Una exposición de estos modelos puede encontrarse, entre otros, en Anselin (1988), Anselin y Hudak (1992) y LeSage y Pace (2009).

${ }^{7}$ El método de Griffith $(1996,2000)$ se ha utilizado con éxito en el campo de la economía: en el análisis de la tasa de desempleo en un contexto espacial (ver Kosfeld y Dreger, 2006; Patuelli et
} 
de Griffith descompone la variable estudiada en función de una componente sistemática o no espacial, que es una combinación lineal de las variables explicativas observadas, y una componente espacial formada por una combinación lineal de variables sintéticas que representan la estructura espacial del conjunto de datos. Estas componentes se pueden incorporar en un marco de modelización de mínimos cuadrados ordinarios (MCO) o de modelos lineales generalizados (MLG). En los trabajos de Griffith $(1996,2000)$ se consideran los autovectores de la matriz $M \cdot W \cdot M$, donde $W$ es la matriz de pesos y $M=I-1\left(1^{\prime} 1\right)^{-1} 1, I$ es la matriz identidad de orden n, y 1 un vector de orden $\mathrm{n} \times 1$ cuyos elementos son todos iguales a 1 ; y la técnica que desarrollan consiste en introducir como regresores estos autovectores para construir un modelo de regresión lineal en el que los errores no presentan dependencia espacial, por lo que se pueden utilizar las técnicas de estimación MCO.

\section{Metodología, cálculo y selección de filtros espaciales}

En este trabajo, la matriz de pesos utilizada $W$ se ha obtenido utilizando el criterio de la distancia mínima y el C-esquema de codificación ${ }^{8}$. De forma resumida, las etapas del filtrado espacial realizado ${ }^{9}$ son:

$1^{\text {a) }}$ Calcular los autovectores de la matriz de distancia transformada $M \cdot W \cdot M$.

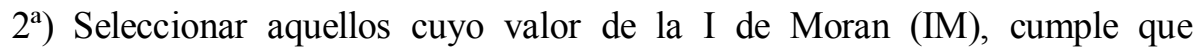
$\mathrm{IM} / \max (\mathrm{IM})$ es mayor o igual que 0,25 (ver Griffith, 2003). El conjunto obtenido está formado por 5 autovectores, que se denotan por $e_{1}, e_{2}, e_{3}, e_{4}, e_{5}$.

$3^{\mathrm{a}}$ ) Considerar como explicativas los autovectores del conjunto obtenido en el paso anterior junto con las variables socioeconómicas que se utilizan como explicativas y suprimir los autovectores que no son significativos. Esto se realiza por medio de una regresión por etapas que se basa en el criterio de información de Akaike (CIA); tal como indican Patuelli et al. (2011), este criterio tiende a sobreseleccionar, por lo que siguiendo a estos autores, después de la regresión por etapas, se realiza una eliminación manual hacia atrás de regresores. Secuencialmente, se elimina una variable en

al., 2011; Buendía y Sánchez, 2013) y en el crecimiento económico (Cravo y Resende, 2013).

${ }^{8}$ Este esquema también conocido como estandarizado se utiliza con mucha frecuencia y consiste en multiplicar los elementos de la matriz básica de partida por $n / D$, siendo $n$ el orden de la matriz (igual al número de observaciones) y $\mathrm{D}$ la suma de todos los elementos de la matriz, la matriz así obtenida es simétrica. Una definición de los esquemas de codificación se encuentra en Tiefelsdorf et al. (1999).

${ }^{9}$ El procedimiento utilizado aquí es similar al que se presenta en Patuelli et al. (2011). 
cada paso hasta que todas las que quedan superan los tests $\chi^{2}$ para un $5 \%$ de significación.

En la Tabla 2 se recogen los autovectores significativos para cada uno de los años considerados, así como los que son comunes. Se obtiene que los autovectores $e_{1}$ y $e_{2}$ son significativos para cada uno de los seis años considerados.

$4^{\text {a) }}$ Tras haber seleccionado los autovectores significativos para todo el periodo, se estima un modelo de panel que incluye el filtro espacial obtenido para capturar la dependencia espacial en todo el periodo ${ }^{10}$.

Tabla 2

Autovectores específicos del año y comunes del periodo 2008-2013

\begin{tabular}{cccccc}
\hline Año & $\begin{array}{c}\text { Número de } \\
\text { autovectores }\end{array}$ & $\begin{array}{c}\text { Autovectores } \\
\text { específicos }\end{array}$ & $\begin{array}{c}\text { Autovectores } \\
\text { comunes }\end{array}$ & $R^{2}$ & AIC \\
\hline 2008 & 2 & & & 0,8837 & 741,59 \\
2009 & 2 & & 0,8787 & 738,03 \\
2010 & 3 & $e_{5}$ & 0,8930 & 729,6 \\
2011 & 3 & $e_{5}$ & $e_{1}, e_{2}$ & 0,8787 & 734,9 \\
2012 & 3 & $e_{5}$ & & 0,8537 & 740,79 \\
2013 & 3 & $e_{5}$ & & 0,8679 & 736,76 \\
\hline
\end{tabular}

Fuente: Elaboración propia.

La idoneidad de utilizar estimaciones econométricas basadas en datos de panel (o datos longitudinales) que consisten en observaciones de corte transversal repetidas a lo largo del tiempo frente al modelo transversal (o temporal), es que al considerar conjuntamente las dos dimensiones, la espacial y la temporal, se incorpora mayor variación al contenido informativo y aumenta el número de grados de libertad por lo que mejora la eficiencia de las estimaciones. En efecto, los modelos de panel realizan un análisis dinámico al incorporar la dimensión temporal de los datos, incluyendo efectos que no pueden ser considerados en los modelos de corte transversal o temporal, lo que enriquece el estudio realizado.

\section{ESTIMACIÓN DEL MODELO A NIVEL PROVINCIAL Y SU EXTRAPOLACIÓN AL ÁMBITO MUNICIPAL}

Como se ha puesto de manifiesto en el desarrollo metodológico, es necesario comprobar si existe un componente espacial en el modelo explicativo del valor

${ }^{10}$ Este procedimiento es utilizado en los trabajos de Griffith (2008) y Patuelli et al.(2011). 
añadido bruto en el ámbito provincial ${ }^{11}$. Para ello, previamente se necesita disponer de las variables seleccionadas. Como ya se mencionó, el proceso de búsqueda y selección de indicadores es crucial en la estimación econométrica y la selección de los indicadores (variables proxy) se realiza exigiendo un doble criterio: primero, dado que la variable dependiente es la renta producida $\left(\mathrm{VAB}_{\mathrm{pc}}\right)$ las variables independientes seleccionadas deben tener un vínculo explicativo fuerte desde la perspectiva teórica con la generación de actividad ${ }^{12}$ (factores productivos) independientemente del nivel de agregación territorial. Segundo, se exige que las variables independientes tengan un comportamiento espacial similar en el ámbito provincial y municipal y que estén disponibles en los dos ámbitos.

Las variables exógenas que cumplen los requisitos anteriores son el número de afiliados a la Seguridad Social y el número de empresas. En efecto, se puede considerar que el número de afiliados a la Seguridad Social es una variable proxy de la utilización del factor productivo trabajo y a su vez el número de empresas se relaciona con el factor capital.

Tabla 3

Variables explicativas y correlaciones, 2008-2013

\begin{tabular}{lc}
\hline \multicolumn{1}{c}{ Variable } & Correlación \\
\hline 1. Afiliados a la Seguridad Social $\left(\mathrm{AFI}_{\mathrm{pc}}\right)$ & 0,82 \\
2. Número de empresas $\left(\mathrm{EMP}_{\mathrm{pc}}\right)$ & 0,58 \\
\hline
\end{tabular}

Fuente: Elaboración propia.

Las variables explicativas utilizadas y su relación con la variable endógena se recogen en la Tabla 3. El número de afiliados a la Seguridad Social es indicativo del nivel de empleo y actividad y, por tanto, de las rentas generadas en un territorio. Asimismo, el número de empresas explica los niveles de actividad generados en cada unidad espacial. Las variables están expresadas en

${ }^{11}$ Los datos de Valor Añadido Bruto (VAB) provincial proceden de la publicación del PIB y sus componentes realizada por el Instituto Nacional de Estadística en 2016. Los datos de las variables explicativas proceden de las estadísticas del Ministerio de Trabajo y Seguridad Social, del Directorio de Empresas del Instituto Nacional de Estadística, de la Agencia Estatal de Administración Tributaria y los servicios de Estadística del País Vasco y la Comunidad Foral de Navarra. Los datos están disponibles en los siguientes enlaces online:

http://www.ine.es/inebmenu/mnu_cuentas.htm

http://www.empleo.gob.es/es/estadisticas/mercado_trabajo/AFI/.htm

http://http://www.agenciatributaria.es/AEAT.internet/Inicio/La_Agencia_Tributaria/Memorias_y _estadisticastributarias/Estadisticas/Estadisticas_por_impuesto/Estadisticas_por_impuesto.shtml

${ }^{12}$ En el caso de la RDBH la selección de indicadores sería diferente al relacionarse con la variable renta disponible y estaría integrada por otros indicadores del tipo número de entidades de depósito, número de automóviles, número de líneas telefónicas, base imponible declarada en el impuesto de la renta de las personas físicas, impuesto del valor añadido, etc. 
términos relativos respecto a la población, ya que presenta la ventaja de evitar problemas de multicolinealidad que son consecuencia de la estrecha relación de los indicadores con el tamaño de la población.

En la Tabla 4 se presentan las correlaciones espaciales en el ámbito provincial y municipal de las variables explicativas, poniendo de manifiesto los valores registrados que la relación entre éstas y el valor añadido bruto no sería muy diferente en el ámbito provincial y municipal ${ }^{13}$.

Tabla 4

Correlaciones entre las variables explicativas 2008-2013

\begin{tabular}{|c|c|c|c|c|c|}
\hline & \multicolumn{2}{|c|}{ Provinciales } & & \multicolumn{2}{|c|}{ Municipales } \\
\hline & AFIpc & EMpc & & AFIpc & EMPpc \\
\hline AFIpc & 1 & 0,61 & AFIpc & 1 & 0,74 \\
\hline EMPpc & 0,61 & 1 & EMPpc & 0,74 & 1 \\
\hline
\end{tabular}

Fuente: Elaboración propia.

Una vez seleccionadas las variables del modelo, se realiza un análisis exploratorio para ver si se observan pautas de asociación espacial. Para contrastar la presencia de autocorrelación espacial, se calcula el estadístico I de Moran ${ }^{14}$ (IM) utilizando la matriz de distancia mínima (WD), estandarizada en la forma descrita anteriormente.

En la Tabla 5 se presentan los resultados obtenidos por el test I de Moran, que indican la existencia de autocorrelación espacial positiva en todas ellas. Como se observa, la intensidad de la dependencia espacial es especialmente elevada en la distribución de la variable endógena, con valores superiores a 0,5 .

Tabla 5

Autocorrelación espacial de las variables según el test I de Moran

\begin{tabular}{lcccccc}
\hline & Matriz & $\boldsymbol{I}$ & Media & S.D. & Z valor & Prob. \\
\hline VAB $_{\text {pc }} \mathbf{0 8}$ & WD & 0,4760 & $-0,02$ & 0,049 & 10,073 & 0,000 \\
VAB $_{\text {pc }}$ 09 & WD & 0,4745 & $-0,02$ & 0,049 & 10,003 & 0,000 \\
VAB $_{\text {pc }} \mathbf{1 0}$ & WD & 0,5073 & $-0,02$ & 0,049 & 10,632 & 0,000 \\
VAB $_{\text {pc }} \mathbf{1 1}$ & WD & 0,5090 & $-0,02$ & 0,049 & 10,681 & 0,000 \\
\hline
\end{tabular}

${ }^{13}$ Como señala Alañón (2002) constatar el supuesto del comportamiento espacial similar de las variables explicativas en los diferentes niveles de agregación es una tarea muy complicada debido a la dificultad de obtener medidas directamente comparables. Las observaciones en el ámbito provincial y municipal son muy diferentes en tamaño y forma por lo que los estadísticos utilizados no serían directamente comparables, además de la dificultad de comparar las interacciones espaciales entre las observaciones a través de las matrices de distancia o contigüidad a escala provincial y municipal.

${ }^{14}$ El estadístico I de Moran es una medida de autocorrelación espacial que nos permite realizar el contraste de autocorrelación espacial. 
Tabla 5 (Continuación)

Autocorrelación espacial de las variables según el test I de Moran

\begin{tabular}{ccccccc}
\hline & Matriz & $\boldsymbol{I}$ & Media & S.D. & Z valor & Prob. \\
\hline $\mathbf{V A B}_{\mathbf{p c}} \mathbf{1 2}$ & WD & 0,4924 & $-0,02$ & 0,049 & 10,345 & 0,000 \\
$\mathbf{V A B}_{\mathbf{p c}} \mathbf{1 3}$ & WD & 0,5006 & $-0,02$ & 0,049 & 10,504 & 0,000 \\
$\mathbf{A F I}_{\mathbf{p c}} \mathbf{0 8}$ & WD & 0,313 & $-0,02$ & 0,049 & 6,735 & 0,000 \\
$\mathbf{A F I}_{\mathrm{pc}} \mathbf{0 9}$ & WD & 0,280 & $-0,02$ & 0,049 & 6,086 & 0,000 \\
$\mathbf{A F I}_{\mathbf{p c}} \mathbf{1 0}$ & WD & 0,282 & $-0,02$ & 0,049 & 6,145 & 0,000 \\
$\mathbf{A F I}_{\mathbf{p c}} \mathbf{1 1}$ & WD & 0,289 & $-0,02$ & 0,049 & 6,281 & 0,000 \\
$\mathbf{A F I}_{\mathbf{p c}} \mathbf{1 2}$ & WD & 0,284 & $-0,02$ & 0,049 & 6,156 & 0,000 \\
$\mathbf{A F I}_{\mathbf{p c}} \mathbf{1 3}$ & WD & 0,292 & $-0,02$ & 0,049 & 6,317 & 0,000 \\
$\mathbf{E M P}_{\mathbf{p c}} \mathbf{0 8}$ & WD & 0,145 & $-0,02$ & 0,049 & 3,365 & 0,000 \\
$\mathbf{E M P}_{\mathbf{p c}} \mathbf{0 9}$ & WD & 0,180 & $-0,02$ & 0,049 & 4,076 & 0,000 \\
$\mathbf{E M P}_{\mathbf{p c}} \mathbf{1 0}$ & WD & 0,204 & $-0,02$ & 0,049 & 4,560 & 0,000 \\
$\mathbf{E M P}_{\mathbf{p c}} \mathbf{1 1}$ & WD & 0,078 & $-0,02$ & 0,043 & 2,267 & 0,020 \\
$\mathbf{E M P}_{\mathbf{p c}} \mathbf{1 2}$ & WD & 0,086 & $-0,02$ & 0,044 & 2,409 & 0,010 \\
$\mathbf{E M P}_{\mathbf{p c}} \mathbf{1 3}$ & WD & 0,097 & $-0,02$ & 0,045 & 2,601 & 0,002 \\
\hline
\end{tabular}

Fuente: Elaboración propia.

En términos del diagrama y mapa de dispersión de Moran aplicado a la propia variable endógena $\mathrm{VAB}_{\mathrm{pc}}$, se observa la existencia de dependencia espacial positiva en los cuadrantes I y III del diagrama (Figura 1), es decir, valores altos de una variable están rodeados de valores altos (color rojo en el mapa) o valores bajos están rodeados de valores bajos (color azul oscuro). En efecto, el elevado coeficiente de la I de Moran y la pendiente positiva del ajuste son indicativos de la presencia de una elevada autocorrelación espacial positiva, con una concentración de valores altos/bajos de las mismas en las diferentes provincias. En general, existe una concentración de valores altos en las provincias del norte/nordeste de España mientras que los niveles más bajos tienden a agruparse en las provincias del sur/suroeste.

Figura 1

Mapa y diagrama de dispersión de la variable endógena

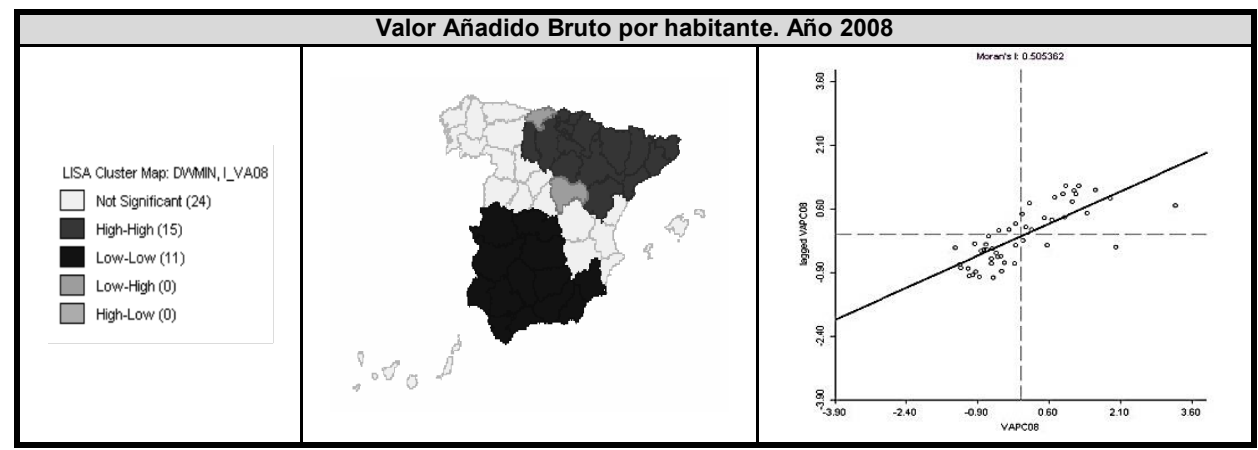


Figura 1 (Continuación)

Mapa y diagrama de dispersión de la variable endógena

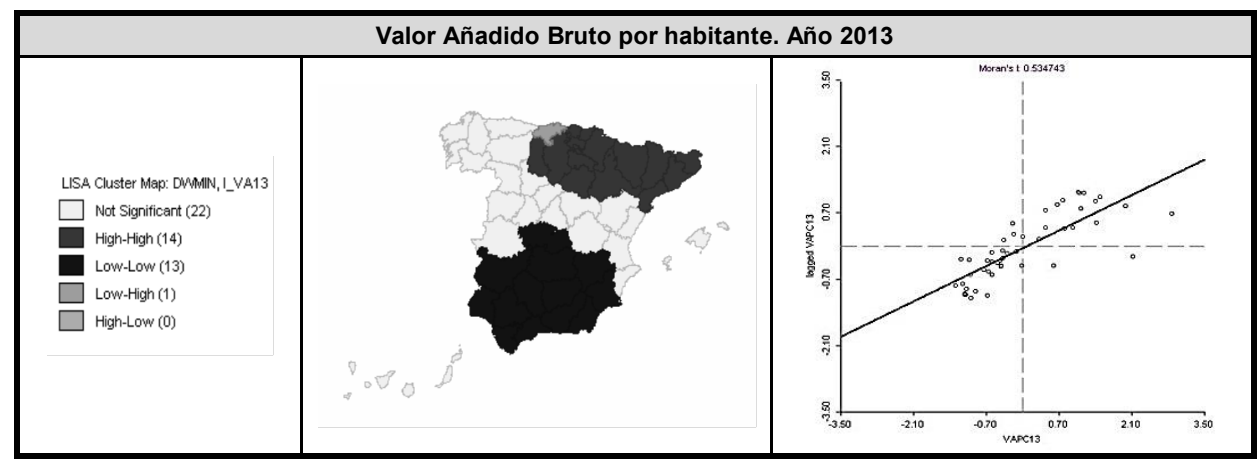

Fuente: Elaboración propia.

En el caso de las variables explicativas, el mapa y diagrama de dispersión (Figura 2) también pone de manifiesto el mismo esquema de dependencia espacial positiva con una concentración de valores altos en el norte y un agrupamiento de valores bajos en las provincias del sur. No obstante, como puede apreciarse en el caso de las variables explicativas el coeficiente de Moran es más reducido y por tanto la asociación espacial en la distribución de las variables en el ámbito provincial es de menor intensidad siendo el número de provincias en las que no se muestra evidencia de autocorrelación espacial mayor que en el caso de la variable dependiente. En la distribución espacial del número de empresas por cada mil habitantes la distancia entre las provincias muestra una menor influencia en la relación de sus valores.

Figura 2

Mapa y diagrama de dispersión de las variables explicativas

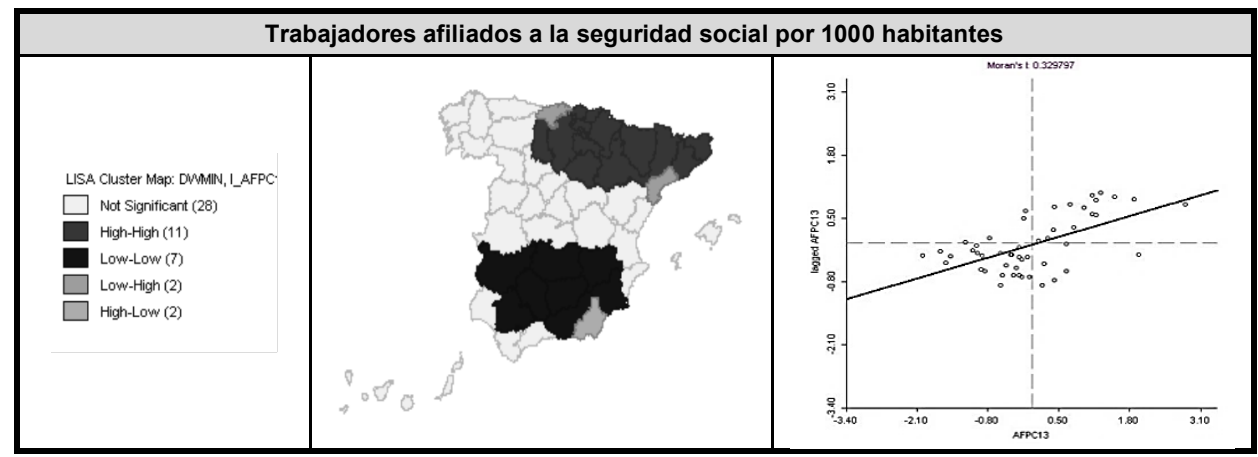


Figura 2 (Continuación)

Mapa y diagrama de dispersión de las variables explicativas

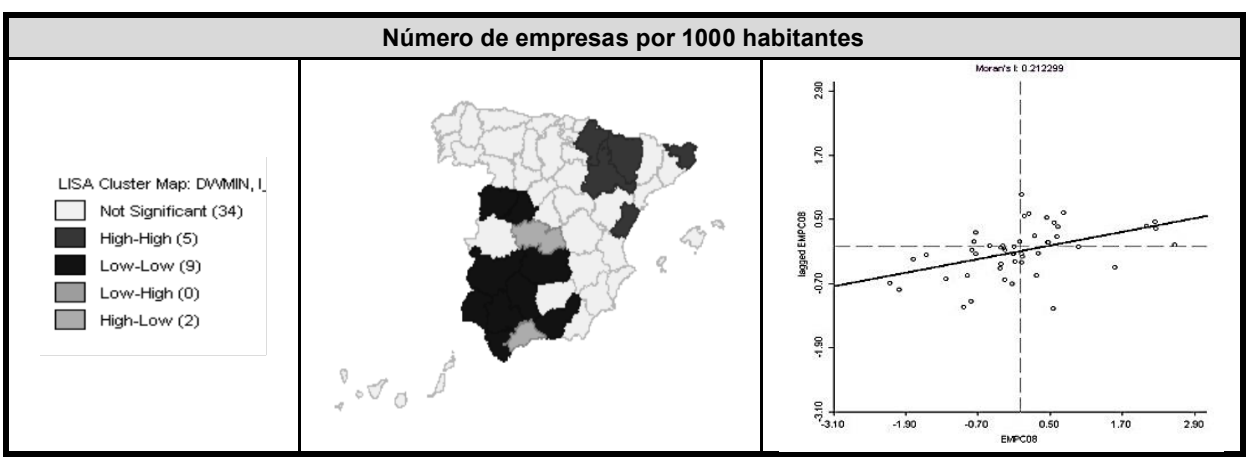

Fuente: Elaboración propia.

Los resultados anteriores ponen de manifiesto la necesidad de incorporar el filtro espacial que capture la dependencia espacial ${ }^{15}$ en el modelo explicativo del valor añadido per cápita.

Definidas todas las variables del modelo y detectada la presencia de autocorrelación espacial, es importante también plantear cómo o de qué forma se introducen dichas variables en la regresión, ya que esto afecta a los resultados de estimación de las rentas provinciales. Es decir, en la medida que no se impone ninguna condición de transversalidad que garantice la igualdad entre la suma de los valores añadidos brutos provinciales y el total nacional, se hace necesario establecer a posteriori un sistema de reparto de la diferencia existente entre las dos variables. Aquí, optamos por incorporar las variables en términos de desviaciones respecto a sus respectivas medias muestrales por lo que para calcular la renta de cada provincia, añadiremos a la desviación estimada el $\mathrm{VAB}_{\mathrm{pc}}$ medio nacional.

Finalmente, se realiza la extrapolación a los municipios de la Región de Murcia de los coeficientes obtenidos en la estimación provincial.

\section{Resultados de la estimación en el ámbito provincial}

La muestra incluye las 50 provincias españolas ${ }^{16}$, excluidas las provincias de Ceuta y Melilla, durante el periodo 2008-2013.

De forma compacta el modelo de panel a estimar se puede expresar en los siguientes términos:

${ }^{15}$ Otra opción metodológica es incorporar el esquema de dependencia en el modelo, mediante la formulación de modelos autorregresivos de dependencia sustantiva o dependencia residual.

${ }^{16}$ Se corresponde con la clasificación NUTS-3 de la Unión Europea. Esta clasificación de región responde más a criterios administrativos que funcionales. 


$$
Y_{i t}=X_{i t} \beta+f e_{i t} \gamma+\varepsilon_{i t}
$$

donde:

$Y_{i t}$ : El valor añadido bruto per cápita de la provincia $\mathrm{i}$ en el periodo $\mathrm{t}$

$X_{i t}$ : Las variables explicativas seleccionadas de la provincia $i$ en el periodo $t$

$f e_{i t}$ : El filtro espacial formado por los autovectores comunes seleccionados

$\varepsilon_{i t}$ : perturbación aleatoria de la provincia i en el periodo $t$.

Por tanto, la especificación econométrica estimada es:

$$
d Y p c_{i t}=\boldsymbol{\beta}_{1} \text { dafipc }_{i t}+\boldsymbol{\beta}_{2} \operatorname{demppc}_{i t}+\boldsymbol{\phi} e_{i t}+\boldsymbol{\varepsilon}_{\mathrm{it}}
$$

donde las variables están expresadas en diferencias respecto a sus valores medios. $d Y p c_{i t}$ es el valor añadido bruto por habitante, dafipc $_{i t}$ es el número de trabajadores afiliados a la Seguridad Social por cada 1000 habitante, demppc $c_{i t}$ es el número de empresas por cada 1000 habitantes, $f e_{i t}$ es una combinación lineal de las componentes del filtro espacial seleccionado y $\varepsilon_{i t}$ es el término de error en la provincia $i$ en el tiempo t.

En la Tabla 6 se presentan conjuntamente los resultados de la estimación de los parámetros de los modelos de panel espacial obtenidos inicialmente con la estimación econométrica por $\mathrm{MCO}^{17}$ [modelo 1], los derivados de la estimación $\mathrm{MV}^{18}$ del modelo autorregresivo de dependencia sustantiva ${ }^{19}$ [modelo 2] y los resultados de la estimación del modelo propuesto de filtrado espacial [modelo 3].

Como se puede observar, la estimación del modelo convencional MCO [Modelo 1] no es válida ya que los valores de los residuos de la I de Moran y de

${ }^{17}$ En el caso de que la I de Moran de los residuos y los contrastes habituales de los multiplicadores de Lagrange (los tests LM-LAG y LM-LE detectan la autocorrelación espacial en la variable dependiente, siendo el segundo robusto ante la existencia de un término de perturbación correlacionado espacialmente, mientras que los tests LM-ERR y LM-EL están diseñados para detectar la autocorrelación espacial en los términos de error, si bien el test LM-EL presenta la ventaja de ser robusto ante posibles especificaciones erróneas locales como la presencia de una variable endógena retardada espacialmente) rechacen la hipótesis nula de ausencia de dependencia espacial, las estimaciones de los parámetros son ineficientes, la varianza residual sesgada y las predicciones ineficientes. Por tanto, ya no son fiables ni las medidas de ajuste ni la inferencia y se incumplen las hipótesis que normalmente se suponen en sus estimadores (insesgadez, mínima varianza y consistencia). Además el coeficiente de determinación $\mathrm{R}^{2}$ estará sobrevalorado.

${ }^{18}$ En presencia de autocorrelación espacial la estimación máximo-verosímil (MV) se presenta como un método de estimación apropiado, en el que se incluye el esquema de dependencia espacial detectado: dependencia sustantiva o dependencia residual.

${ }^{19}$ Una exposición de los modelos de panel espacial puede encontrarse en Elhorst (2010). La estimación del modelo 2 se ha realizado usando el software desarrollado por Elhorst (2014). 
los contrastes del multiplicador de Lagrange (LM) ponen de manifiesto la existencia de dependencia espacial. El valor del contraste LM-LAG es mayor que el de LM-ERR lo que indica la existencia de un esquema de dependencia sustantiva, siendo confirmado por los valores de las formas robustas de estos contrastes puesto que el test LM-LE es significativo mientras que el LM-EL no.

Por tanto, se puede reespecificar el modelo incluyendo el esquema de dependencia espacial mediante el retardo espacial de la variable dependiente [Modelo 2], cuyo valor es positivo $\rho=0,39$ y altamente significativo. El enfoque alternativo al tratamiento de la autocorrelación espacial [Modelo 3] obtiene unos resultados más satisfactorios (menor porcentaje de error respecto a los valores observados) en el que todas las variables explicativas son altamente significativas y los signos de sus coeficientes son los que cabe esperar. Estos resultados confirman también la notable importancia relativa del componente espacial en la explicación de la distribución del valor añadido per cápita.

\section{Tabla 6}

Estimación de los parámetros de los modelos de panel espacial. 2008-2013

\begin{tabular}{|c|c|c|c|}
\hline & $\begin{array}{c}\text { [Modelo 1] } \\
\text { Estimación MCo } \\
d Y_{i t}=\beta_{1} d a f i p c_{i t}+\beta_{2} d e m p p c_{i t}+\varepsilon_{i t}\end{array}$ & $\begin{array}{c}\text { [Modelo 2] } \\
\text { Dependencia sustantiva } \\
d Y_{i t}=\rho W d Y_{i t}+\beta_{1} d a f i p c_{i t}+\beta_{2} \operatorname{dempp}_{c_{i t}}+\varepsilon_{\mathrm{it}}\end{array}$ & $\begin{array}{c}\text { [Modelo 3] } \\
\text { Filtrado espacial } \\
d Y_{i t}=\beta_{1} d a f i p c_{i t}+\beta_{2} \text { demppc }_{i t}+\varphi f_{e_{i t}}+\varepsilon_{\mathrm{it}}\end{array}$ \\
\hline$\overline{D A F I} I_{p c}$ & $\begin{array}{c}79,143^{\star \star *} \\
(0,0000)\end{array}$ & $\begin{array}{c}25,49^{\star \star \star} \\
(0,000)\end{array}$ & $\begin{array}{c}28,77^{\star \star *} \\
(0,000)\end{array}$ \\
\hline $\mathrm{DEMP}_{\mathrm{pc}}$ & $\begin{array}{l}91,918^{*} \\
(0,0302)\end{array}$ & $\begin{array}{l}18,09^{* *} \\
(0,004)\end{array}$ & $\begin{array}{c}18,47^{* * *} \\
(0,000)\end{array}$ \\
\hline$e_{1}$ & & & $\begin{array}{c}13588,8^{* * *} \\
(0,000)\end{array}$ \\
\hline$e_{2}$ & & & $\begin{array}{c}10942,9^{* * *} \\
(0,000)\end{array}$ \\
\hline $\operatorname{CAE}^{\mathrm{a}}(\rho)$ & & $\begin{array}{c}0,3939^{* * *} \\
(0,0000)\end{array}$ & \\
\hline $\mathrm{R}^{2}$ & 0,7541 & 0,6896 & 0,6310 \\
\hline Jarque-Bera & $\begin{array}{l}0,23793 \\
(0,8878)\end{array}$ & & \\
\hline Breusch-Pagan & $\begin{array}{l}12,281^{* * *} \\
(0,0004)\end{array}$ & & \\
\hline I-Moran & $\begin{array}{c}0,1898^{\star * *} \\
(0,0047)\end{array}$ & & \\
\hline LM-ERR & $\begin{array}{c}38,7429^{\star \star *} \\
(0,0000)\end{array}$ & & \\
\hline LM-EL & $\begin{array}{c}0,6006^{* * *} \\
(0,4380)\end{array}$ & & \\
\hline LM-LAG & $\begin{array}{c}117.6722^{* * *} \\
(0,0000)\end{array}$ & & \\
\hline LM-LE & $\begin{array}{c}79,1338^{\star \star \star} \\
(0,0000)\end{array}$ & & \\
\hline
\end{tabular}

Entre paréntesis el p-valor de los estadísticos t-Student (MCO) o z (MV).

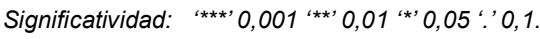

${ }^{a}$ Coeficiente de autocorrelación espacial.

Fuente: Elaboración propia. 
En la Tabla 7 se presentan los porcentajes de error de la estimación provincial realizada según el modelo de dependencia espacial [Modelo 2] y el modelo de filtrado espacial [Modelo 3] permitiéndonos realizar la comparativa entre ambos modelos ${ }^{20}$. Como se observa, sistemáticamente el porcentaje de error del valor estimado es sistemáticamente superior en el modelo de dependencia sustantiva [Modelo 2].

Globalmente, el modelo de filtrado espacial [Modelo 3] a nivel provincial sobrevalora un $0,8 \%$ el VAB total respecto al valor observado ${ }^{21}$. Si analizamos los errores provinciales observamos que hay cinco provincias con errores superiores al 30\% y cuatro con errores superiores al 20\%. En general, la estimación tiende a sobrevalorar el valor observado puesto que este resultado se produce en 34 de las 50 provincias. Cuando consideramos la dimensión económica de las provincias, se constata que de las 16 grandes $^{22}$ en 7 de ellas se subestima el valor observado mientras que en las consideradas pequeñas en 24 de las 34 provincias se subestima dicho valor. Los mayores porcentajes de error están asociados a estimaciones que sobrevaloran el valor observado. Por tanto, el modelo tiende a sobreestimar el $\mathrm{VAB}_{\mathrm{pc}}$ pero sin ningún patrón definido en cuanto a las características de las provincias. En el caso de la Región de Murcia (RM) se sobreestima el valor observado en un $17,2 \%$.

Tabla 7

Comparación del porcentaje de error en la estimación provincial, 2008-2013

\begin{tabular}{lcc}
\hline & [Modelo 2] & [Modelo 3] \\
\hline Álava & $-25,2$ & $-25,0$ \\
Albacete & 19,0 & 18,8 \\
Alicante & 17,2 & 16,8 \\
Almería & 24,0 & 23,9 \\
Ávila & 14,4 & 14,1 \\
Badajoz & 37,0 & 36,6 \\
Baleares & $-2,9$ & $-2,8$ \\
Barcelona & $-8,6$ & $-8,3$ \\
Burgos & $-9,8$ & $-9,7$ \\
Cáceres & 37,2 & 36,9 \\
Cádiz & 18,1 & 17,4 \\
Castellón & 2,6 & 2,5 \\
Ciudad Real & 11,4 & 11,0 \\
\hline
\end{tabular}

${ }^{20}$ Las desviaciones del modelo 1 no son consideradas puesto que la estimación no es válida en presencia de dependencia espacial.

${ }^{21}$ Las desviaciones globales que se producen para cada uno de los años son del 2,4\%; 1,9\%; 1,6\%; $2,6 \% ; 0 \%, 1,8 \%$ y $0 \%$, respectivamente. Solamente en el año 2011 el valor estimado infravalora el valor observado. El modelo 2 se obtiene una desviación global muy similar del 0,9\%.

${ }^{22}$ Bajo esta clasificación se consideran aquellas provincias con una participación mayor o igual al $2 \%$ del VAB total. En el caso de las dos más grandes Madrid y Barcelona se subestima el valor observado. 
Tabla 7 (Continuación)

Comparación del porcentaje de error en la estimación provincial, 2008-2013

\begin{tabular}{|c|c|c|}
\hline & [Modelo 2] & [Modelo 3] \\
\hline Córdoba & 34,0 & 33,8 \\
\hline Coruña & 5,8 & 5,8 \\
\hline Cuenca & 15,0 & 14,8 \\
\hline Girona & $-11,3$ & $-11,2$ \\
\hline Granada & 30,9 & 30,7 \\
\hline Guadalajara & 5,1 & 4,7 \\
\hline Guipúzcoa & $-17,1$ & $-16,7$ \\
\hline Huelva & 30,1 & 29,8 \\
\hline Huesca & $-8,2$ & $-8,1$ \\
\hline Jaén & 36,7 & 36,3 \\
\hline León & 7,3 & 7,1 \\
\hline Lleida & $-11,0$ & $-10,8$ \\
\hline La Rioja & $-6,0$ & $-6,0$ \\
\hline Lugo & 16,6 & 16,5 \\
\hline Madrid & $-19,3$ & $-19,1$ \\
\hline Málaga & 25,9 & 25,6 \\
\hline Murcia & 17,4 & 17,2 \\
\hline Navarra & $-15,6$ & $-15,5$ \\
\hline Ourense & 11,8 & 11,6 \\
\hline Asturias & 5,8 & 5,6 \\
\hline Palencia & $-5,1$ & $-5,2$ \\
\hline Palmas (Las) & 10,5 & 10,3 \\
\hline Pontevedra & 15,5 & 15,4 \\
\hline Salamanca & 13,3 & 13,1 \\
\hline $\begin{array}{l}\text { Santa Cruz de } \\
\text { Tenerife }\end{array}$ & 9,2 & 8,9 \\
\hline Cantabria & 4,8 & 4,7 \\
\hline Segovia & 7,7 & 7,7 \\
\hline Sevilla & 18,5 & 18,3 \\
\hline Soria & 2,9 & 2,9 \\
\hline Tarragona & $-15,5$ & $-15,5$ \\
\hline Teruel & $-7,2$ & $-7,3$ \\
\hline Toledo & 19,5 & 19,2 \\
\hline Valencia & 4,9 & 4,8 \\
\hline Valladolid & 0,9 & 0,9 \\
\hline Vizcaya & $-16,5$ & $-16,4$ \\
\hline Zamora & 14,9 & 14,6 \\
\hline Zaragoza & $-6,2$ & $-6,1$ \\
\hline
\end{tabular}

Fuente: Elaboración propia.

En resumen, con este nuevo enfoque econométrico en la estimación del $\mathrm{VAB}_{\mathrm{pc}}$ municipal que contempla la heterogeneidad espacial y la autocorrelación espacial en los valores provinciales del $\mathrm{VAB}_{\mathrm{pc}}$ se utiliza el filtro espacial como sustituto de los efectos fijos en el marco de estimación de panel. La ventaja que proporciona la utilización del filtro espacial es que al incorporar información específica de la provincia que genera autocorrelación espacial libera grados de libertad y corrige la autocorrelación espacial en los residuos. No obstante, 
aunque este enfoque proporciona el mejor ajuste (menor porcentaje de error) respecto a los otros modelos no está exento de limitaciones en la medida que no todas las observaciones presentan autocorrelación espacial de la variable dependiente afectando a la información sobre los patrones espaciales en las provincias y los resultados estimados.

\section{Aplicación al ámbito municipal}

Una vez realizada la regresión y estimados los coeficientes, estos se aplican a las correspondientes variables municipales expresadas en forma de desviaciones, de tal forma que se obtiene para cada municipio la desviación de su renta per cápita respecto a la renta per cápita provincial.

Su expresión formal es:

$$
\hat{y}_{m}=\sum_{j=1}^{K} \hat{\beta}_{j} x_{j m} m=1,2, \ldots, M
$$

donde las variables en minúscula $\hat{y}_{m}$ y $x_{j m}$ representan las desviaciones de la renta per cápita y de las variables explicativas, respectivamente; $\mathrm{M}$ es el número de municipios y $\hat{\beta}_{j}$ los coeficientes estimados de las variables.

En la expresión [3] se verifica que:

$$
\hat{y}_{m}=\hat{Y}_{m}-\bar{Y} \quad ; \quad x_{j m}=X_{j m}-\bar{X}_{j}
$$

siendo:

$\hat{Y}_{m}:$ VAB per cápita estimado del municipio $\mathrm{m}$

$\bar{Y}:$ VAB per cápita provincial

$X_{j m}:$ Indicador per cápita j-ésimo del municipio $\mathrm{m}$

$\bar{X}_{j}$ : Indicador per cápita j-ésimo provincial

Como puede observarse, el VAB por habitante de cada municipio se obtiene en la expresión [4] despejando $\hat{Y}_{m}$, es decir, el VAB por habitante del municipio $\mathrm{m}$ se obtiene añadiendo al $\mathrm{VAB}$ por habitante provincial $\bar{Y}$ la desviación correspondiente $\hat{y}_{m}$

Finalmente, hay que ajustar el valor añadido estimado de los municipios al del conjunto de la Región de Murcia, para lo que definimos $V$, VAB de la Región de Murcia; $V_{i}$, VAB del municipio i y $P_{i}$ la población del municipio i.

El VAB total del municipio i estimado $\hat{V}_{i}$ será: $\hat{V}_{i}=P_{i} \hat{Y}_{i}$. 
El VAB total estimado de la Región de Murcia $\hat{V}$ será: $\hat{V}=\sum_{i=1}^{M} \hat{V}_{i}$, donde $\mathrm{M}$ es el número de municipios de la región.

Por tanto, el factor de ajuste es $\delta=\frac{V}{\hat{V}}$, y el valor añadido bruto per cápita del municipio i será: $Y_{i}=\delta \hat{Y}_{i} \quad i=1, \ldots ., M$.

Los resultados obtenidos de la distribución del $\mathrm{VAB}_{\mathrm{pc}}$, tanto en valores absolutos como en porcentaje respecto al promedio correspondientes a los años 2008 y 2013, están contenidos en la Tabla 8. Algunos de los aspectos más relevantes de los resultados obtenidos son, en primer lugar la existencia de una elevada concentración del VAB (47,5\%) en las dos aglomeraciones urbanas ${ }^{23}$ más importantes de la RM, Murcia (34,2\%) y Cartagena (13,3\%). Los municipios de Lorca (6,5\%), Molina de Segura (4,5\%), Alcantarilla (2,5\%), Torre-Pacheco $(2,5 \%)$, Yecla $(2,3 \%)$ y Totana $(2,2 \%)$ concentran el $20,5 \%$ del $\mathrm{VAB}$ y el $20,4 \%$ de la población. En segundo lugar, los municipios con mayor $\mathrm{VAB}_{\mathrm{pc}}$ en 2013 son Lorquí (20.804 €), Alhama de Murcia (19.279 €) y Murcia $(19.012 €)$. En tercer lugar, atendiendo a la distribución relativa de la $\mathrm{RDB}_{\mathrm{pc}}$ por municipios, los que alcanzan una renta superior a la media regional en 2008 y 2013 (renta muy alta y alta) son los de Lorquí, Alhama de Murcia, Murcia, Torre Pacheco, Fuente Álamo, Totana, Campos del Río, Lorca y Yecla. En lado opuesto, los municipios que registran un $\mathrm{VAB}_{\mathrm{pc}}$ significativamente inferior a la media regional en los dos años extremos (renta muy baja y baja) son Ricote, Ulea, Villanueva del Río Segura, Ojós, Albudeite, Pliego, La Unión, Alguazas, Cehegín y Moratalla. Estos diez municipios en el año 2013 concentran el 3,27\% del $\mathrm{VAB}$ regional y el $4,34 \%$ de la población, en una superficie que representa el $13,56 \%$ del total.

En la Tabla 9 se presentan los resultados obtenidos expresados en términos relativos respecto del $\mathrm{VAB}_{\mathrm{pc}}$ medio regional, el crecimiento de las variables $\mathrm{VAB}_{\mathrm{pc}}, \mathrm{VAB}$ y la población, así como el ranking y la distancia respecto al municipio con mayor $\mathrm{VAB}$ por habitante. Esta distancia se obtiene mediante el cociente entre el $\mathrm{VAB}_{\mathrm{pc}}$ municipal mayor y el $\mathrm{VAB}_{\mathrm{pc}}$ de cada municipio y es indicativa del crecimiento que debería experimentar la renta del municipio para alcanzar la renta máxima. Como puede apreciarse, la elevada polarización (ocho municipios representan el 39,5\% de la superficie y acumulan el $68 \%$ del $\mathrm{VAB}$ y el $65,3 \%$ de la población) es muy persistente durante el periodo de crisis, manteniéndose prácticamente los mismos niveles de concentración (en 2008 el VAB y la población concentrada en estos municipios fue del $68,1 \%$ y $65,6 \%$, respectivamente).

23 En términos de superficie y población en 2013 representaban el $12,8 \%$ y el 44,6\%, respectivamente. En 2008 concentraban el 44,9\% de la población y el 47,5\% del VAB. 
Tabla 8

Valor Añadido Bruto por habitante municipal. 2008 y 2013

\begin{tabular}{|c|c|c|c|c|c|}
\hline Código & Municipio & $\begin{array}{c}\text { VABpc }(€) \\
2008\end{array}$ & $\begin{array}{c}\text { VABpc } \\
\text { Media }=100\end{array}$ & $\begin{array}{c}\text { VABpc }(€) \\
2013\end{array}$ & $\begin{array}{c}\text { VABpc } \\
\text { Media }=100\end{array}$ \\
\hline 1 & Abanilla & 15.790 & 84,1 & 13.333 & 80,7 \\
\hline 2 & Abarán & 17.903 & 95,4 & 16.016 & 96,9 \\
\hline 3 & Águilas & 17.169 & 91,5 & 15.608 & 94,4 \\
\hline 4 & Albudeite & 12.630 & 67,3 & 11.720 & 70,9 \\
\hline 5 & Alcantarilla & 16.885 & 89,9 & 14.938 & 90,4 \\
\hline 6 & Aledo & 14.990 & 79,8 & 14.442 & 87,4 \\
\hline 7 & Alguazas & 14.818 & 78,9 & 12.250 & 74,1 \\
\hline 8 & Alhama & 20.547 & 109,4 & 19.280 & 116,7 \\
\hline 9 & Archena & 15.952 & 85,0 & 14.390 & 87,1 \\
\hline 10 & Beniel & 16.355 & 87,1 & 14.190 & 85,9 \\
\hline 11 & Blanca & 17.372 & 92,5 & 14.526 & 87,9 \\
\hline 12 & Bullas & 16.712 & 89,0 & 13.742 & 83,2 \\
\hline 13 & Calasparra & 17.080 & 91,0 & 14.722 & 89,1 \\
\hline 14 & Campos del Río & 19.073 & 101,6 & 17.125 & 103,6 \\
\hline 15 & Caravaca & 17.467 & 93,0 & 16.178 & 97,9 \\
\hline 16 & Cartagena & 16.772 & 89,3 & 14.846 & 89,8 \\
\hline 17 & Cehegín & 15.020 & 80,0 & 13.591 & 82,2 \\
\hline 18 & Ceutí & 17.124 & 91,2 & 14.851 & 89,9 \\
\hline 19 & Cieza & 16.362 & 87,2 & 14.755 & 89,3 \\
\hline 20 & Fortuna & 17.277 & 92,0 & 15.689 & 94,9 \\
\hline 21 & Fuente Álamo & 20.792 & 110,7 & 17.941 & 108,6 \\
\hline 22 & Jumilla & 17.152 & 91,4 & 14.574 & 88,2 \\
\hline 23 & Librilla & 16.739 & 89,2 & 14.327 & 86,7 \\
\hline 24 & Lorca & 19.116 & 101,8 & 17.124 & 103,6 \\
\hline 25 & Lorquí & 23.802 & 126,8 & 20.804 & 125,9 \\
\hline 26 & Mazarrón & 17.072 & 90,9 & 14.162 & 85,7 \\
\hline 27 & Molina de Segura & 18.048 & 96,1 & 15.959 & 96,6 \\
\hline 28 & Moratalla & 15.533 & 82,7 & 13.916 & 84,2 \\
\hline 29 & Mula & 16.095 & 85,7 & 14.126 & 85,5 \\
\hline 30 & Murcia & 21.333 & 113,6 & 19.012 & 115,0 \\
\hline 31 & Ojós & 12.557 & 66,9 & 12.953 & 78,4 \\
\hline 32 & Pliego & 13.259 & 70,6 & 11.990 & 72,5 \\
\hline 33 & Puerto Lumbreras & 17.837 & 95,0 & 15.646 & 94,7 \\
\hline 34 & Ricote & 12.033 & 64,1 & 10.939 & 66,2 \\
\hline 35 & San Javier & 18.929 & 100,8 & 15.706 & 95,0 \\
\hline 36 & San Pedro & 16.739 & 89,2 & 14.413 & 87,2 \\
\hline 37 & Torre-Pacheco & 23.933 & 127,5 & 18.278 & 110,6 \\
\hline 38 & Torres de Cotillas & 17.957 & 95,6 & 16.123 & 97,6 \\
\hline 39 & Totana & 19.878 & 105,9 & 17.700 & 107,1 \\
\hline 40 & Ulea & 12.433 & 66,2 & 10.666 & 64,5 \\
\hline 41 & Unión (La) & 13.906 & 74,1 & 11.463 & 69,4 \\
\hline 42 & Villanueva del RS & 12.551 & 66,9 & 11.426 & 69,1 \\
\hline 43 & Yecla & 19.475 & 103,7 & 16.601 & 100,4 \\
\hline 44 & Santomera & 19.053 & 101,5 & 16.296 & 98,6 \\
\hline \multirow[t]{2}{*}{45} & Alcázares & 16.006 & 85,3 & 13.819 & 83,6 \\
\hline & Región de Murcia & 18.774 & 100 & 16.527 & 100 \\
\hline
\end{tabular}

Fuente: Elaboración propia. 
Tabla 9

Crecimiento y distribución municipal del VABpc, 2008-2013

\begin{tabular}{|c|c|c|c|c|c|c|c|c|c|}
\hline \multirow[b]{3}{*}{ Código } & \multirow[b]{3}{*}{ Municipio } & \multicolumn{3}{|c|}{$\mathrm{RDBH}_{\mathrm{pc}}$ relativo } & \multirow{2}{*}{\multicolumn{3}{|c|}{ TMAA (\%) }} & \multirow{2}{*}{\multicolumn{2}{|c|}{ Distancia }} \\
\hline & & \multicolumn{3}{|c|}{ Ranking } & & & & & \\
\hline & & 2008 & 2013 & 2013 & VABpc & VAB & Población & 2008 & 2013 \\
\hline 1 & Abanilla & 84,1 & 80,7 & 37 & $-3,3$ & $-3,6$ & $-0,2$ & 1,52 & 1,56 \\
\hline 2 & Abarán & 95,4 & 96,9 & 13 & $-2,2$ & $-1,9$ & 0,3 & 1,34 & 1,30 \\
\hline 3 & Águilas & 91,5 & 94,4 & 18 & $-1,9$ & $-1,4$ & 0,5 & 1,39 & 1,33 \\
\hline 4 & Albudeite & 67,3 & 70,9 & 41 & $-1,5$ & $-1,6$ & $-0,1$ & 1,89 & 1,78 \\
\hline 5 & Alcantarilla & 89,9 & 90,4 & 19 & $-2,4$ & $-2,3$ & 0,1 & 1,42 & 1,39 \\
\hline 6 & Aledo & 79,8 & 87,4 & 26 & $-0,7$ & $-1,7$ & $-1,0$ & 1,62 & 1,44 \\
\hline 7 & Alguazas & 78,9 & 74,1 & 39 & $-3,7$ & $-2,3$ & 1,5 & 1,16 & 1,70 \\
\hline 8 & Alhama & 109,4 & 116,7 & 2 & $-1,3$ & 0,5 & 1,8 & 1,50 & 1,08 \\
\hline 9 & Archena & 85,0 & 87,1 & 28 & $-2,0$ & $-1,9$ & 0,1 & 1,46 & 1,45 \\
\hline 10 & Beniel & 87,1 & 85,9 & 30 & $-2,8$ & $-1,8$ & 1,1 & 1,38 & 1,47 \\
\hline 11 & Blanca & 92,5 & 87,9 & 25 & $-3,5$ & $-2,8$ & 0,7 & 1,43 & 1,43 \\
\hline 12 & Bullas & 89,0 & 83,2 & 35 & $-3,8$ & $-4,0$ & $-0,1$ & 1,40 & 1,51 \\
\hline 13 & Calasparra & 91,0 & 89,1 & 23 & $-2,9$ & $-2,9$ & 0,0 & 1,25 & 1,41 \\
\hline 14 & Campos del Río & 101,6 & 103,6 & 7 & $-2,1$ & $-2,5$ & $-0,3$ & 1,37 & 1,21 \\
\hline 15 & Caravaca & 93,0 & 97,9 & 11 & $-1,5$ & $-1,7$ & $-0,2$ & 1,43 & 1,29 \\
\hline 16 & Cartagena & 89,3 & 89,8 & 21 & $-2,4$ & $-1,7$ & 0,7 & 1,59 & 1,40 \\
\hline 17 & Cehegín & 80,0 & 82,2 & 36 & $-2,0$ & $-1,9$ & 0,1 & 1,40 & 1,53 \\
\hline 18 & Ceutí & 91,2 & 89,9 & 20 & $-2,8$ & $-0,5$ & 2,4 & 1,46 & 1,40 \\
\hline 19 & Cieza & 87,2 & 89,3 & 22 & $-2,0$ & $-2,0$ & 0,1 & 1,39 & 1,41 \\
\hline 20 & Fortuna & 92,0 & 94,9 & 16 & $-1,9$ & $-1,2$ & 0,7 & 1,15 & 1,33 \\
\hline 21 & Fuente Álamo & 110,7 & 108,6 & 5 & $-2,9$ & $-0,7$ & 2,2 & 1,40 & 1,16 \\
\hline 22 & Jumilla & 91,4 & 88,2 & 24 & $-3,2$ & $-2,9$ & 0,3 & 1,43 & 1,43 \\
\hline 23 & Librilla & 89,2 & 86,7 & 29 & $-3,1$ & $-1,4$ & 1,7 & 1,25 & 1,45 \\
\hline 24 & Lorca & 101,8 & 103,6 & 8 & $-2,2$ & $-1,8$ & 0,4 & 1,01 & 1,21 \\
\hline 25 & Lorquí & 126,8 & 125,9 & 1 & $-2,7$ & $-2,5$ & 0,1 & 1,40 & 1,00 \\
\hline 26 & Mazarrón & 90,9 & 85,7 & 31 & $-3,7$ & $-2,9$ & 0,8 & 1,33 & 1,47 \\
\hline 27 & Molina de Segura & 96,1 & 96,6 & 14 & $-2,4$ & $-0,6$ & 1,9 & 1,54 & 1,30 \\
\hline 28 & Moratalla & 82,7 & 84,2 & 33 & $-2,2$ & $-2,6$ & $-0,5$ & 1,49 & 1,49 \\
\hline 29 & Mula & 85,7 & 85,5 & 32 & $-2,6$ & $-2,4$ & 0,1 & 1,12 & 1,47 \\
\hline 30 & Murcia & 113,6 & 115,0 & 3 & $-2,3$ & $-1,9$ & 0,4 & 1,91 & 1,09 \\
\hline 31 & Ojós & 66,9 & 78,4 & 38 & 0,6 & $-1,9$ & $-2,5$ & 1,80 & 1,61 \\
\hline 32 & Pliego & 70,6 & 72,5 & 40 & $-2,0$ & $-2,6$ & $-0,7$ & 1,34 & 1,74 \\
\hline 33 & Puerto Lumbreras & 95,0 & 94,7 & 17 & $-2,6$ & $-1,3$ & 1,4 & 1,99 & 1,33 \\
\hline 34 & Ricote & 64,1 & 66,2 & 44 & $-1,9$ & $-3,6$ & $-1,7$ & 1,26 & 1,90 \\
\hline 35 & San Javier & 100,8 & 95,0 & 15 & $-3,7$ & $-2,4$ & 1,4 & 1,43 & 1,32 \\
\hline 36 & San Pedro & 89,2 & 87,2 & 27 & $-2,9$ & $-2,3$ & 0,7 & 1,00 & 1,44 \\
\hline 37 & Torre-Pacheco & 127,5 & 110,6 & 4 & $-5,2$ & $-3,3$ & 2,0 & 1,20 & 1,14 \\
\hline 38 & Torres de C & 95,6 & 97,6 & 12 & $-2,1$ & $-1,1$ & 1,1 & 1,93 & 1,29 \\
\hline 39 & Totana & 105,9 & 107,1 & 6 & $-2,3$ & $-1,3$ & 1,0 & 1,72 & 1,18 \\
\hline 40 & Ulea & 66,2 & 64,5 & 45 & $-3,0$ & $-3,4$ & $-0,4$ & 1,91 & 1,95 \\
\hline 41 & Unión (La) & 74,1 & 69,4 & 42 & $-3,8$ & $-1,5$ & 2,4 & 1,23 & 1,81 \\
\hline 42 & Villanueva del RS & 66,9 & 69,1 & 43 & $-1,9$ & 0,4 & 2,3 & 1,26 & 1,82 \\
\hline 43 & Yecla & 103,7 & 100,4 & 9 & $-3,1$ & $-3,4$ & $-0,3$ & 1,50 & 1,25 \\
\hline 44 & Santomera & 101,5 & 98,6 & 10 & $-3,1$ & $-2,0$ & 1,1 & 1,33 & 1,28 \\
\hline \multirow[t]{2}{*}{45} & Alcázares & 85,3 & 83,6 & 34 & $-2,9$ & $-1,2$ & 1,8 & 1,60 & 1,51 \\
\hline & Región de Murcia & 100 & 100 & & $-2,6$ & $-2,0$ & 0,6 & 1,52 & 1,26 \\
\hline
\end{tabular}

Fuente: Elaboración propia. 
$\mathrm{Si}$ atendemos a la dinámica del $\mathrm{VAB}_{\mathrm{pc}}, \mathrm{VAB}$ y población durante el periodo contemplado, se observa que en la RM el descenso ha sido más acusado que en el ámbito nacional en términos de $\mathrm{VAB}$ y $\mathrm{VABpc}$, registrando tasas negativas del $-2,5 \%$ y $-1,9 \%$, con un diferencial mayor de 0,4 y 0,2 puntos porcentuales, respectivamente. En cambio, la población ha experimentado un crecimiento del $0,6 \%$, frente al $0,4 \%$ nacional. Por otro lado, atendiendo a los valores iniciales y finales de la distribución municipal del $\mathrm{VAB}_{\mathrm{pc}}$ en los dos años 2008 y 2013, se constata que la distancia entre los municipios de mayor y menor renta se reduce levemente desde 1,99 veces en 2008 hasta 1,95 veces en 2013, y no hay cambios significativos en el orden de los mismos.

Finalmente, hay que destacar que todos los municipios experimentan caídas del $\mathrm{VAB}_{\mathrm{pc}}$. No obstante, de los veintidós municipios cuyo descenso es superior a la media $(2,5 \%)$, solo once obtienen crecimientos de la población muy superiores al respectivo valor medio $(0,6 \%)$.

Figura 3

Valor Añadido Bruto por habitante municipal en la Región de Murcia, 2008 y 2013

\begin{tabular}{|l}
\hline \\
Rents
\end{tabular}

Fuente: Elaboración propia.

Por último, atendiendo a la distribución municipal de la dinámica recesiva experimentada por todos los municipios, hay que constatar la elevada estabilidad que han mantenido la mayoría de los municipios en cuanto a su posición relativa (Figura 3). Como se puede observar, solo once municipios experimentan cambios en sus posiciones relativas. Los municipios que empeoran su posición relativa en el periodo 2008-2013 son Los Alcázares (renta media a renta baja), Alguazas 
(renta baja a renta muy baja), Bullas (renta media a renta baja), San Javier (renta alta a renta media), Santomera (renta alta a renta media) y Torre-Pacheco (renta muy alta a renta alta). Por el contrario, los que experimentan una mejora relativa son Aledo (renta baja a renta media), Archena (renta baja a renta media), Ojós (renta muy baja a renta baja), Alhama de Murcia (renta alta a renta muy alta) y Murcia (renta alta a renta muy alta).

\section{CONCLUSIONES}

1. Actualmente un procedimiento idóneo para realizar estimaciones de renta en el ámbito municipal mediante un método indirecto debiera contemplar la características de los datos y fenómenos espaciales, en particular la presencia de autocorrelación o dependencia espacial y utilizar la metodología de los datos de panel. En este trabajo, se ha estimado el valor añadido bruto por habitante de las provincias españolas mediante un modelo de panel siguiendo el procedimiento del filtrado semiparamétrico de Griffith $(1996,2000)$ que descompone la variable estudiada en función de una componente sistemática o no espacial y una componente espacial.

2. Se ha verificado que el mejor ajuste lo proporciona el modelo de panel con el filtrado semiparamétrico de Griffith frente a las alternativas del modelo espacial de retardo o el tradicional MCO obteniendo el menor porcentaje de error respecto a los valores observados en las diferentes provincias. El modelo utilizado para la estimación del $\mathrm{VAB}_{\mathrm{pc}}$ a nivel provincial tiende a sobrevalorar en un $1 \%$ el valor observado durante el periodo 2008-2013.

3. El principal problema de la estimación de la renta municipal es la aceptación del supuesto del comportamiento espacial similar de las variables explicativas en los diferentes niveles de agregación (provincial, municipal) y la extrapolación de los parámetros estimados en un ámbito territorial a otro distinto. Es el conocido problema de la unidad de área modificable en su vertiente del problema de inferencia ecológica. Esta limitación difícilmente superable puede ser mitigada con la búsqueda de indicadores que expliquen la variable dependiente desde la perspectiva de la teoría económica.

4. El procedimiento seguido de introducir en el modelo las variables en términos de desviaciones respecto a sus respectivas medias muestrales y calcular la renta de cada territorio añadiendo a la desviación estimada la renta media permite ajustar los resultados municipales eliminando el sesgo de la ausencia de la condición de transversalidad.

5. Finalmente, se ha realizado el ejercicio de inferencia ecológica (extrapolación de los parámetros obtenidos en la estimación provincial) a los municipios de la Región de Murcia, obteniéndose unos resultados bastante interesantes sobre el patrón de distribución espacial del VABpc durante el periodo recesivo 2008-2013, en el que cabe subrayar la elevada polarización del VAB y la 
población, la elevada y persistente brecha registrada entre los municipios y la estabilidad y escasa movilidad de los mismos en la distribución espacial de la renta.

6. Los resultados anteriores justifican la utilización de la metodología del filtrado espacial en el marco de datos de panel con fines predictivos de datos microterritoriales que permiten realizar un primer diagnóstico de la situación económica y social en el ámbito municipal.

\section{REFERENCIAS BIBLIOGRÁFICAS}

AGENCIA ESTATAL DE ADMINISTRACIÓN TRIBUTARIA (2016). Memorias y estadísticas tributarias. Dirección on line: http://http://www.agenciatributaria.es/ AEAT.internet/Inicio/La_Agencia_Tributaria/Memorias_y_estadisticastributarias/Estadis ticas/Estadisticas_por_impuesto/Estadisticas_por_impuesto.shtml_

ALAÑóN, A. (2001). La renta regional en España: análisis y estimación de sus determinantes. Tesis doctoral. Universidad Complutense de Madrid.

ALAÑóN, A. (2002). "Estimación del valor añadido de los municipios españoles en 1991 mediante técnicas de econometría espacial". Economiaz, 51, pp. 172-193.

ANSELIN, L. (1988). Spatial econometrics: Methods and models. Dordrecht, Boston: Kluwer Academic Publishers.

ANSELIN, L. y HUDAK, S. (1992). "Spatial econometrics in practice: A review of software options". Regional Science and Urban Economics, 22(3), pp. 509-536.

APARICIO, M.; MARTÍNEZ, C.; SANSO, M. y TRÍVEZ, J. (1983). “Una metodología para la estimación de la renta disponible municipal". VIII Reunión de Estudios Regionales. Bilbao.

BEYAERT, A.; BUENDÍA, J.D. y ESTEBAN, M. (1993). "Distribución intrarregional de la renta". En Colino, J. (dir.): Estructura económica de la Región de Murcia (pp. 715-738). Madrid: Ed. Civitas.

BUENDÍA, J.D. y CALVO-FLORES, A. (1999). Informe sobre la distribución intermunicipal de la renta. Disparidades intermunicipales de la Región de Murcia durante el periodo 1986-1996. Murcia: Consejo Económico y Social de la Región de Murcia.

BUENDÍA, J.D., CALVO-FLORES, A., ESTEBAN, M., y SÁNCHEZ, J.C.(2000). La distribución intermunicipal de la renta en el periodo 1986-1996: estimación, descripción y análisis. Murcia: Consejo Económico y Social de la Región de Murcia.

BUENDÍA, J.D.; CALVO-FLORES, A.; ESTEBAN, M. y SÁNCHEZ, J.C. (2010). Estimación de la renta disponible bruta de los hogares de los municipios de la Región de Murcia durante el periodo 2000-2006. Análisis de las disparidades intermunicipales de renta y bienestar. Murcia: Consejo Económico y Social de la Región de Murcia.

BUENDÍA, J.D. y SÁNCHEZ, M.M. (2013). "La distribución del desempleo en las provincias españolas: Un análisis con datos de panel mediante el filtrado espacial". Investigaciones Regionales, 27, pp.143-154. 
CASTELLS, A.; GUELL, X. y PARELLADA, M. (1982). "Estimació de la renda i el diposits de les comarques i els principals municipis de Catalunya". Revista Económica, 66. Banca Catalana.

CASTELLS, A.; GUELL, X. y PARELLADA, M. (1983). Estimació de la renda de les comarques i els principals municipis de Catalunya. Diputació de Barcelona.

CHASCO, C. (2003). Econometría espacial aplicada a la predicción-extrapolación de datos espaciales. Madrid: Comunidad de Madrid.

CHASCO, C. y LÓPEZ, F. (2004). "Modelos de regresión espacio-temporales en la estimación de la renta municipal: el caso de la región de Murcia". Estudios de Economía Aplicada, 22 (3), pp.1-24.

CRAVO, T.A., y MENDES, G. (2013). "Economic growth in Brazil: a spatial filtering approach". The Annals of Regional Science, 50(2), pp. 555-575.

ELHORST, J.P. (2010). "Spatial Panel Data Models". In Fischer M.M. and Getis A (Eds.): Handbook of Applied Spatial Analysis, (pp. 377-407). Springer: Berlin Heidelberg New York.

ELHORST, J. P. (2014). "Matlab Software for Spatial Panels". International Regional Science Review, 37(3), pp. 389-405.

ESTEBAN, J. y PEDREÑO, A. (1988). "Renta municipal de la Comunidad Autónoma de la Región de Murcia". Cuadernos de Investigación. Alicante: Caja de Ahorros de Alicante y Murcia.

ENRIQUE REGUEIRA, I. (2009). Estimación municipal del valor añadido bruto en Andalucía. Sevilla: Instituto de Estadística de Andalucía. Consejería de Economía y Hacienda.

GRIFFITH, D.A. (1996). "Spatial autocorrelation and eigenfunctions of the geographic weights matrix accompanying geo-referenced data". The Canadian Geographer, 40, pp. 351-367.

GRIFFITH, D.A. (2000). "A linear regression solution to the spatial autocorrelation problem". Journal of Geographical Systems, 2, pp. 141-156.

GRIFFITH, D.A. (2003). Spatial autocorrelation and spatial filtering: gaining understanding through theory and scientific visualization. Berlin: Springer-Verlag,

GRIFFITH, D.A. (2008). "A comparison of four model specifications for describing small heterogeneous space-time datasets: Sugar cane production in Puerto Rico, 1958/591973/74". Papers in Regional Science, 87, pp. 341-55.

INSTITUTO NACIONAL DE ESTADÍSTICA (2016). Contabilidad Regional de España. Dirección on line: http://www.ine.es/inebmenu/mnu_cuentas.htm

KOSFELD, R., y DREGER, C. (2006). "Thresholds for employment and unemployment: A spatial analysis of German regional labour markets, 1992-2000". Papers in Regional Science, 85(4), pp. 523-42.

LESAGE, J. y PACE, R.(2009). Introduction to Spatial Econometrics. CRC Press Inc.

MINISTERIO DE TRABAJO Y SEGURIDAD SOCIAL (2016): Mercado de trabajo. Dirección on line: http://www.empleo.gob.es/es/estadisticas/mercado_trabajo/ index.htm

LÓPEZ, M. y MONTES, F. (1974). Renta comarcal. Informe económico regional 197273. Cámaras de Comercio, Industria y Navegación de Alicante, Alcoy, Castellón, Orihuela y Valencia.

MORAN, P. A. P. (1950). "Notes on Continuous Stochastic Phenomena". Biometrika, 37(1), pp. 17-23. 
MUNS, J. (1971). "La renta de Barcelona ciudad y su zona de influencia. Un intento de determinación". Revista Económica de Banca Catalana, 23.

ORTAS-RICO, M. y HONRUBIA, J. (2014). Renta personal de los municipios españoles y su distribución: Metodología de estimación a partir de microdatos tributarios. Doc. 12. Fundación de Estudios de Economía Aplicada (FEDEA).

OTERO, J.M. y FERNÁNDEZ, A. (1999). La renta de los municipios de Andalucía. Metodología y resultados para 1994. Sevilla: Instituto de Estadística de Andalucía.

PATUELLI, R., GRIFFITH, D., TIEFELSDORF, M. y NIJKAMP, P. (2011). "Spatial filtering and eigenvector stability: space-time models for German unemployment data". International Regional Science Review, 34(2), pp. 253-280.

REMÍREZ, J. A. (1991). Una estimación de la renta familiar disponible a nivel municipal. El caso de Navarra. Madrid: Confederación Española de Cajas de Ahorro.

SERVICIO DE ESTUDIOS DE LA CAIXA (2014). Anuario Económico de España. Caixa. Dirección on line: http://www.anuarieco.lacaixa.comunicacions.com

SOCIEDAD ASTURIANA DE ESTUDIOS ECONÓMICOS E INDUSTRIALES, SADEI (varios años). La renta de los municipios asturianos. Oviedo: Caja de Ahorros de Asturias.

TIEFELSDORF, M.; GRIFFITH, D.A. y BOOTS, B. (1999). "A variance-stabilizing coding scheme for spatial link matrices". Environment and Planning A, 31, pp. 165-180.

VAYÁ, E.; LÓPEZ-BAZO, E.; MORA, A. y SURIÑACH, J. (1996). "Se ven afectados los resultados sobre convergencia regional cuando se consideran los efectos espaciales?". XXII Reunión de Estudios Regionales. 